\title{
THE MODEL FOR LUMINESCENCE CENTER EXCITATION IN $\mathrm{PbWO}_{4}{ }^{*}$
}

\author{
D. Millers, L. Grigorjeva, S. Chernov and V. Pankratov \\ Institute of Solid State Physics, University of Latvia \\ 8 Kengaraga str., 1063, Riga, Latvia
}

(Received July 29, 1998; revised version February 4, 1999)

The luminescence and transient absorption of $\mathrm{PbliO}_{4}$ crystals were studied. The electron beam ( $10 \mathrm{~ns}, E \approx 270 \mathrm{keV}$ ) was used as excitation source. The kinetics of luminescence is complicated - the prompt xise of luminescence intensity is followed by a slow $\left(\tau_{\mathrm{r}} \approx 100 \mathrm{~ns}\right)$ rise. The luminescence decay at $80 \mathrm{~K}$ is nearly exponential with $\tau_{\mathrm{d}} \approx 4.0-4.5 \mu \mathrm{s}$. The decay kinetics of transient absorption at $3.5 \mathrm{eV}$ can be described roughly by two exponents with time constants $\tau_{1} \approx 100 \mathrm{~ns}$ and $\tau_{2} \approx 4.0 \mu \mathrm{s}$. A common process responsible for absorption relaxation and luminescence rise is suggested. The luminescence center excitation occurs during recombination process via $\mathrm{Pb}^{2+}$ excited state formation and subsequent energy transfer to $\mathrm{WO}_{4}^{2-}$ group.

PACS numbers: 78.40.- $\mathrm{q}, 78.60 . \mathrm{Ya}, 61.72 . \mathrm{Cc}$

\section{Introduction}

Scintillation characteristics and the optical properties of $\mathrm{PbWO}_{4}(\mathrm{PWO})$ single crystals have been intensively studied during recent years because PWO satisfy the requirements for modern scintillation detectors used in high energy physics [1-3].

The luminescence spectrum of PWO contains two overlapping bands - a blue $(\approx 3.0 \mathrm{eV})$ and a green $(\approx 2.5 \mathrm{eV})$ - attributed to the regular $\mathrm{WO}_{4}^{2-}$ and distorted $\mathrm{WO}_{3}$ lattice site respectively [1]. Thus, the luminescence centers are tungstate-oxygen complexes, however $\mathrm{Pb}^{2+}$ ions play an essential role in luminescence centers formation $[1,4]$.

The blue luminescence of PWO can be excited via two mechanisms [5]: (1) excitonic and (2) electron-hole (recombination). Two mechanisms of blue luminescence excitation were established from experiments in which PWO was irradiated by pulsed electron beam at low temperature $(80 \mathrm{~K})$ : luminescence pulse rise

*The results of this paper were initially presented at The Jablonski Centennial Conference on Luminescence and Photophysics, July 29-27, 1998, Toruń, Poland. 
front showed two components, a prompt and a delayed. The delayed component $\left(\tau_{\mathrm{d}} \approx 100 \mathrm{~ns}\right)$ of luminescence pulse rise front was attributed to the thermal release of electrons from some trap and subsequent recombination with hole trapped at cation $\left(\mathrm{Pb}^{3+}\right.$ center $)$ as well as anion $\left(\mathrm{O}^{-}\right)$site.

The radiation induced absorption spectra of stable defects at room temperature was studied for PWO crystals [6]. Absorption bands around 2.9 and $3.5 \mathrm{eV}$ were ascribed to hole $\mathrm{O}^{-}$and $\mathrm{Pb}^{3+}$ centers respectively, however the exact interpretation of the nature of hole centers was not, because ESR signals were not observed [7]. Thus, the transient absorption spectra (TAS) study and comparison of kinetics for absorption relaxation with those for luminescence rise and decay can be useful for understanding processes of luminescence center excited state formation.

\section{Experimental}

The PWO crystals for study were obtained from two sources - Bogoroditsk and China. Irradiation source was a pulsed electron beam, pulse width $\approx 10 \mathrm{ns,}$ energy of electrons $270 \mathrm{keV}$. The luminescence and transient absorption measurements were carried out. For luminescence measurements the emission from the sample was collected on the entrance slit of grating monochromator (MDR-2), detected by photomultiplier tube with biallali photocathode and displayed on the storage oscilloscope. The xenon flash lamp as probe light source was used for transient absorption measurements. Probe light was directed on the samples surface irradiated through the samples side, totally reflected from it and came out from the opposite side of the sample. Then the probe light was collected on the entrance slit of monochromator. Registration was the same as for luminescence. The time resolution was 20 ns.

\section{Results and discussion}

The spectra of luminescence of PWO excited by pulsed electron beam are showed in Fig. 1. The spectra at $80 \mathrm{~K}$ at the end of irradiation pulse and delayed for $4 \mu \mathrm{s}$ are showed in Fig. 1a; the spectra measured at $290 \mathrm{~K}$ at the end of irradiation pulse, delayed for $100 \mathrm{~ns}$, and under steady-state X-ray irradiation are showed in Fig. 1b. One can see from Fig. 1a that the luminescence spectrum at $80 \mathrm{~K}$ contained mainly the blue luminescence band, while the green luminescence band was so weak that its contribution was not observed. It is known that the decay of green luminescence is slower than that of blue. Thus the shape of spectrum has to depend on time. The observed luminescence spectrum at $80 \mathrm{~K}$ does not depend on time: during $4 \mu \mathrm{s}$ after irradiation pulse the shape of spectrum was the same as under irradiation pulse. Thus the contribution of green luminescence was very weak at $80 \mathrm{~K}$. Similar result was observed by Tamulaitis et al. for PWO under photoexcitation $\left(E_{\mathrm{exc}}=4.66 \mathrm{eV}\right)$ at $77 \mathrm{~K}[8]$. On the other hand, the luminescence spectrum at $290 \mathrm{~K}$ depends on time: at the end of irradiation pulse the blue luminescence was dominant (Fig. 1b), whereas after 100 ns delay as well as under steady-state X-ray irradiation a strong band of green $(\approx 2.5 \mathrm{eV})$ luminescence was observed. This change of spectrum occurs since the decay or formation time 
of green luminescence is slower than that of blue luminescence. One can point out the similar differences of luminescence decay times at $80 \mathrm{~K}$ are known from experiments under selective photoexcitation [9], however in our experiments under pulsed electron beam irradiation only the blue luminescence was observed. These facts lead to conclusion that energy transfer to the green luminescence centers is not effective at low temperatures.

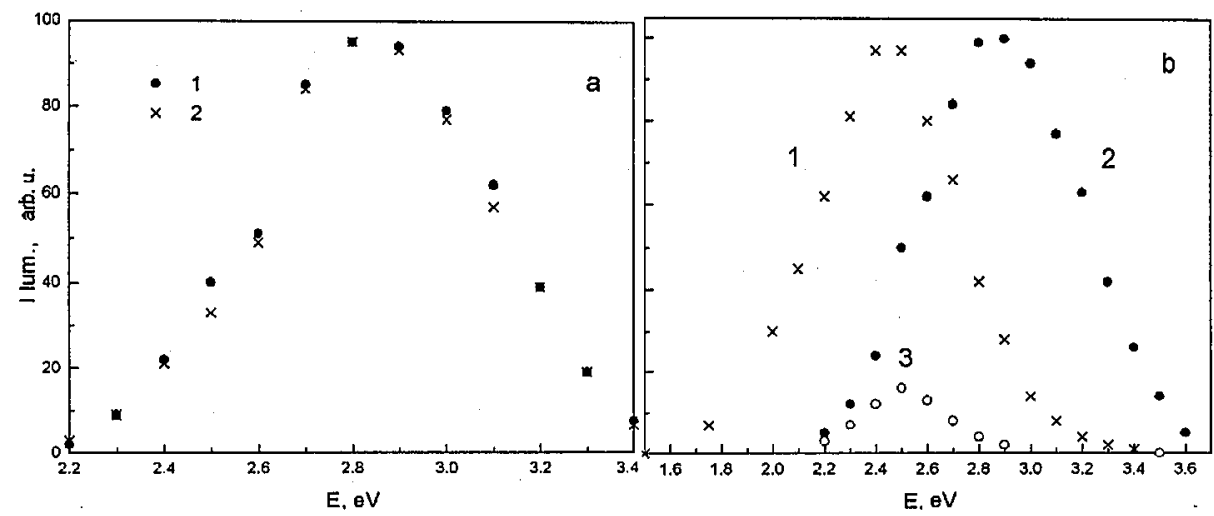

Fig. 1. Normalized luminescence spectra of $\mathrm{PbWO}_{4}$ crystal excited by electron pulse at $80 \mathrm{~K}(\mathrm{a})$ and $\mathrm{RT}(\mathrm{b})$ : (a) at the end of irradiation pulse -1 ; at $4 \mu \mathrm{s}$ delay -2 ; (b) steady state X-ray irradiation -1 ; at the end of irradiation pulse -2 ; at $100 \mathrm{~ns}$ delay -3 .

We suggest that low efficiency of energy transfer by excitons and i ctron-hole pairs to the lattice defects at $80 \mathrm{~K}$ was due to the self-trapping of the electronic excitations. The possibility of self-trapping at least of one kind of charge carriers in PWO is discussed in [10].

The blue luminescence of $\mathrm{PWO}$ was attributed to the radiative annihilation of self-trapped exciton by several authors [8,11]. The hole can be trapped at either oxygen $\left(\mathrm{O}^{-}\right.$center) or lead $\left(\mathrm{Pb}^{3+}\right.$ center $)$. The hole trapped at oxygen near impurity was observed in $\mathrm{ZnWO}_{4}[12]$, whereas self-trapped hole forming $\mathrm{Pb}^{3+}$ center was observed in $\mathrm{PbF}_{2}, \mathrm{PbCO}_{3}$, and $\mathrm{BaPbF}_{6}$ [13]. Thus we suggest that two types of the self-trapped holes in $\mathrm{PWO}$ are possible - as $\mathrm{O}^{-}$center and as $\mathrm{Pb}^{3+}$ center.

The holes as well as electrons became mobile if the temperature was above the delocalization temperature and these mobile holes and electrons can be retrapped by lattice defects. It seems that holes become mobile above $100-130 \mathrm{~K}$ in PWO, because in this region glow peaks showed only the green luminescence [14] - the mobile holes were trapped at $\mathrm{WO}_{3}$ complex which is responsible for the green luminescence. The similar process is valid if irradiation of PWO is performed above temperature of holes self-trapping and results of our experiments demonstrate it (Fig. 1a,b).

The hole centers in PWO have well-resolved absorption bands at $3.5 \mathrm{eV}$ and $2.9 \mathrm{eV}$ ascribed to $\mathrm{Pb}^{3+}$ and $\mathrm{O}^{-}$centers, respectively [6]. Thus we measure the 
transient absorption spectra after irradiation pulse at different delay times as well as absorption relaxation kinetics at different photon energies to determine the hole centers which take part in luminescence center formation.

The induced transient absorption spectra of PWO at the end of irradiation pulse and delayed for $150 \mathrm{~ns}$ at $80 \mathrm{~K}$ are shown in Fig. 2. The spectra reveal three bands located at $3.5 \mathrm{eV}, 2.3 \mathrm{eV}$ and below $1.0 \mathrm{eV}$. The band at $3.5 \mathrm{eV}$ may be due to self-trapped hole at lead, e.g. $\mathrm{Pb}^{3+}$ centers [6], however the absorption of these centers was studied only a little. The transient absorption band peaking at $2.3 \mathrm{eV}$ as well as other observed in our experiments below $1.0 \mathrm{eV}$ may be due to. some electron centers.

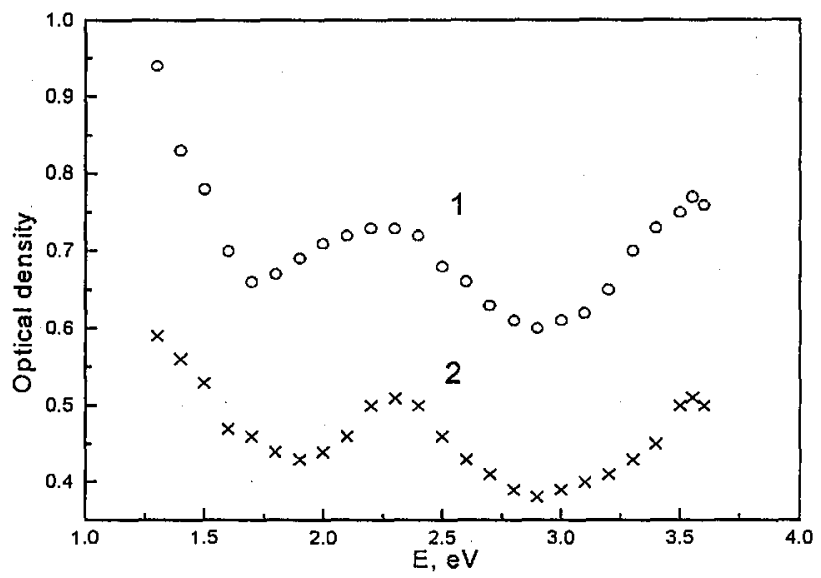

Fig. 2. Transient absorption spectra of $\mathrm{PbWO}_{4}$ crystal under electron pulse irradiation at 80 K. 1 - at the end of irradiation pulse; 2 - at 150 ns delay.

The recombination process in PWO shows the same blue luminescence spectrum and kinetics as it was observed by direct $\mathrm{Pb}^{2+}$ excitation with photons. We suggest that electron trapping at the self-trapped hole leads to the redistribution of the clensity of electron states and creation of $\left(\mathrm{Pb}^{2+}\right)^{*}$ as a result.

The relaxation of transient absorption at $80 \mathrm{~K}$ was nearly the same over whole spectrum. The delayed spectrum in Fig. 2 has the same shape as the initial one. The relaxation kinetics of transient absorption roughly can be approximated by two exponents. Figure 3 shows the kinetics at $3.4 \mathrm{eV}$, and from these kinetics time constants $\tau_{1} \approx 90-95$ ns and $\tau_{2} \approx 4 \mu$ s were calculated.

The pulse of luminescence was observed as a response on irradiation by electron beam pulse. Shape of blue luminescence pulse at $80 \mathrm{~K}$ was studied in [5]. The delayed (related to irradiation pulse end) rise front of luminescence pulse was observed. The luminescence rise front is exponential and at $80 \mathrm{~K}$ time constant is $\tau_{\mathrm{r}} \approx 90 \mathrm{~ns}$. This time constant is the same as $\tau_{1}$ for transient absorption relaxation at $80 \mathrm{~K}$. Thus the luminescence rise and transient absorption relaxation was caused by the same process and this process is thermally induced escape of electrons from some trap followed by retrapping at the hole trapped center. It can be pointed out that transient absorption spectra contain the band at $3.5 \mathrm{eV}$ ascribed to $\mathrm{Pb}^{3+}$ 


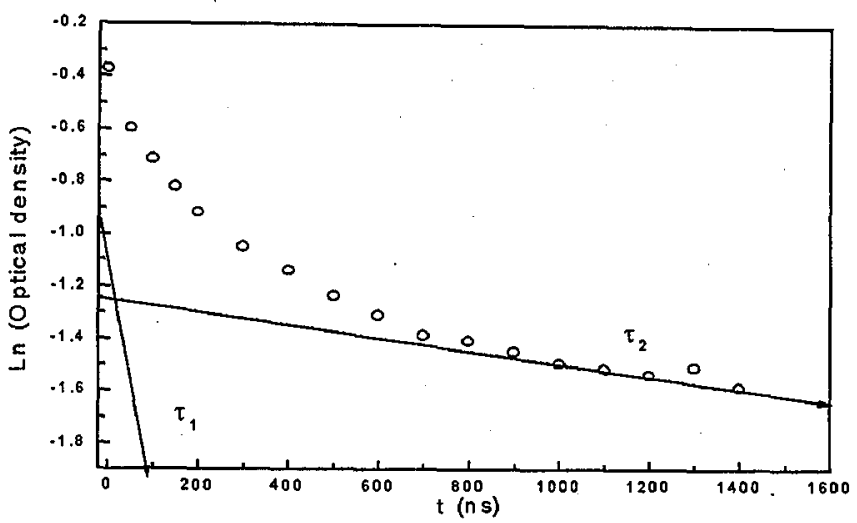

Fig. 3. The relaxation kinetics of transient absorption $\mathrm{PbWO}_{4}$ crystals at $80 \mathrm{~K}$ after electron pulse irradiation.

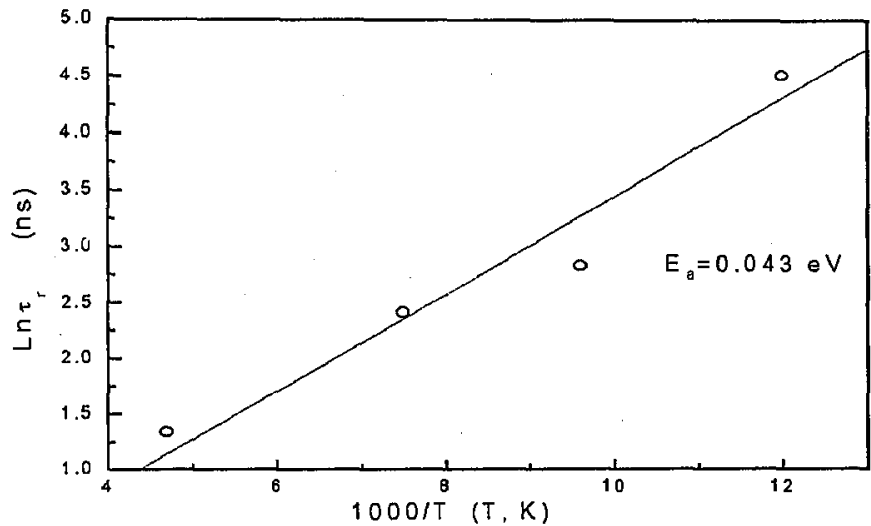

Fig. 4. The temperature dependence of luminescence rise time.

center and do not reveal a band at $2.9 \mathrm{eV}$ ascribed to $\mathrm{O}^{-}$center (Fig. 2). Thus we conclude that electron trapping at $\mathrm{Pb}^{3+}$ center takes place. This process results in formation of excited state $\left(\mathrm{Pb}^{2+}\right)^{*}$. The next step is energy transfer from $\left(\mathrm{Pb}^{2+}\right)^{*}$ to neighboring $\mathrm{WO}_{4}^{2-}$ group and radiative decay of $\left(\mathrm{WO}_{4}^{2-}\right)^{*}$ gives rise to the blue luminescence.

The dependence of luminescence rise time on temperature is shown in Fig. 4. The activation energy estimated from this dependence is $0.04 \mathrm{eV}$ which is very close to $0.05 \mathrm{eV}$ estimated from thermostimulated luminescence (TSL) peak at $28 \mathrm{~K}$ [14]. Thus, the same shallow electron trap is responsible for luminescence pulse rise front and fast decay of induced absorption observed in our experiments and for glow peak at $28 \mathrm{~K}$. The extrapolation of the data from Fig. 4 to $290 \mathrm{~K}$ gives a luminescence rise time constant 1.5-2.0 ns. It seems that this process limited the shortest scintillation in PWO at room temperature. 


\section{Conclusions}

Transient absorption spectra of PWO reveals bands due to hole centers, associated with $\mathrm{Pb}$ states and electron traps responsible for TSL peak at $28 \mathrm{~K}$.

The transient absorption relaxation fast component time constant and blue luminescence pulse rise front time constant coincide at $80 \mathrm{~K}$. The electronic states of lead are involved in the blue luminescence excitation during recombination process. The luminescence center appears as the result of electron detrapping from the shallow trap, followed by trapping on $\mathrm{Pb}^{3+}$ center and subsequent transfer of energy to the tungstate group.

\section{References}

[1] P. Lecoq, I. Dafinei, A. Auffray, M. Schneegans, M.V. Korzik, O.V. Missevitch, V.P. Pavlenko, A.A. Fedorov, A.N. Annenkov, V.L. Kostylev, V.D. Ligun, Nucl. Instrum. Methods Phys. Res. A 365, 291 (1995).

[2] M. Martini, G. Spinolo, A. Vedda, M. Nikl, K. Nitsch, V. Hamplova, P. Fabeni, G.P. Pazzi, I. Dafinei, P. Lecoq, Chem. Phys. Lett. 260, 418 (1996).

[3] R.Y. Zhu, D.A. Ma, H.B. Newman, C.L. Woody, J.A. Kierstead, S.P. Stoll, P.W. Levy, Nucl. Instrum. Methods Phys. Rev. A 376, 319 (1996).

[4] A.N. Belsky, V.V. Mikhailin, A.N. Vasil'ev, I. Dafinei, P. Lecoq, C. Pedrini, P. Chevallier, P. Dhez, P. Martin, Chem. Phys. Lett. 243, 552 (1995).

[5] D. Millers, L. Grigorjeva, S. Chernov, A. Popov, P. Lecoq, E. Auffray, Phys. Status Solidi B 203, 585 (1997).

[6] M. Nikl, K. Nitsch, S. Baccaro, A. Cecilia, M. Montecchi, B. Borgia, I. Dafinei, M. Diemoz, M. Martini, E. Rosetta, G. Spinola, A. Vedda, M. Kobayashi, M. Ishii, Y. Usuki, O. Jarolimek, P. Reiche, J. Appl. Phys. 82, 5758 (1997).

[7] A. Hofstaetter, R. Oeder, A. Scharmann, D. Shwabe, B. Vitte, Phys. Status Solidi $B$ 89, 375 (1998).

[8] G. Tamulaitis, S. Buračas, V.P. Martinov, V.D. Ryzhikov, H.H. Gutbrod, V.I. Manko, Phys. Status Solidi A 157, 187 (1996).

[9] M. Nikl, K. Nitsch, K. Polak, E. Mihokova, I. Dafinei, E. Auffray, P. Lecoq, P. Reiche, R. Uecker, G.P. Pazzi, Phys. Status Solidi B 195, 331 (1996).

[10] M. Nikl, P. Bohaček, K. Nitsch, E. Mihokova, M. Martini, A. Vedda, S. Croci, G.P. Pazzi, P. Fabeni, S. Baccaro, B. Borgia, I. Dafinei, M. Diemoz, G. Organtini, E. Auffrai, P. Lecoq, M. Kobayashi, M. Ishii, Y. Usuki, Appl. Phys. Lett. 71, 26, 3755 (1997).

[11] D.M. Bordun, J. Appl. Spectrosc. 65, N1, 145 (1998).

[12] A. Watterich, A. Hofstaetter, Solid State Commun. 105, 357 (1998).

[13] S.V. Nistor, D. Schoemaker, I. Ursu, Phys. Status Solidi B 185, 9 (1994).

[14] M. Springis, V. Tale, I. Tale, J. Lumin. 72-74, 784 (1997). 